$\mathrm{p}=0.0123, \mathrm{HR}=3.029 ; 95 \% \mathrm{CI}:$ 1.4-6.5). Median time from $\mathrm{OC}$ diagnosis to $\mathrm{BM}$ and from disease recurrence to $\mathrm{BM}$, was longer for BRCA+ compared to BRCA-(44.3mo vs. $32.3 \mathrm{mo}$ and $11.8 \mathrm{mo}$ vs. $0.7 \mathrm{mo}$, respectively). Median survival(mOS) was not significantly different in patients with BM or without $\mathrm{BM}(59.4 \mathrm{mo}$ vs. $71.2 \mathrm{mo}, \mathrm{p}=0.36)$. Following diagnosis of $\mathrm{BM}$, mOS was $20.6 \mathrm{mo}$ among BRCA+ and $12.3 \mathrm{mo}$ among BRCA $(p=0.4266)$. No correlation was found with PARP inhibitors or bevacizumab treatment.

Conclusion* BM are an infrequent among OC patients. However, the risk is three-folds higher among BRCA+. Interestingly, BM do not significantly alter survival among OC patients; might be related to longer survival in BRCA+ or higher tropism or else.

\section{CONCORDANCE OF THE FDA-APPROVED COMPANION DIAGNOSTIC AND A NEXT-GENERATION SEQUENCING ASSAY KIT FOR ASSESSING HOMOLOGOUS RECOMBINATION DEFICIENCY IN OVARIAN CANCER}

${ }^{1} \mathrm{~W}$ Weichert*, ${ }^{2} \mathrm{P}$ Qiu, ${ }^{2} \mathrm{~J}$ Lunceford, ${ }^{2} \mathrm{~A}$ Wehn, ${ }^{3} \mathrm{~A}$ Yarunin, ${ }^{2} \mathrm{M}$ Marton. 'Institute of Pathology, Technical University of Munich, Munich, Germany; ${ }^{2}$ Merck and Co., Inc., Kenilworth, USA; ${ }^{3}$ AstraZeneca, Alderley Park, UK

\subsection{6/ijgc-2021-ESG0.665}

Introduction/Background* Olaparib+bevacizumab is approved as first-line maintenance treatment of advanced homologous recombination deficiency (HRD)-positive ovarian cancer (OC), defined by the presence of a deleterious or suspected deleterious BRCA mutation (BRCAm) and/or genomic instability (evaluated with a United States Food and Drug Administrationapproved companion diagnostic). We evaluated the performance of an in-development next-generation sequencing assay, based on Illumina's RUO TSO 500 content, that identifies variants in tumour tissue and HRD genomic scars (Illumina test). Herein, we report the performances of the in-development Illumina test versus the Myriad myChoice PLUS assay (Myriad test).

Methodology OC tissue samples were analysed with Illumina $(n=227 ; 40$ ng DNA) and Myriad tests $(n=254 ; 200 n g$ DNA). Samples that failed QC during the first run using the Illumina test were retested with higher DNA input. Agreement rates for BRCAm, genomic instability score (GIS), and HRD status (includes BRCA and GIS [cutoff, 42]) were analysed. For the overall and the non-BRCAm cohorts, correlation between the continuous GIS of the Illumina and Myriad tests was determined. The analytical sensitivity and specificity of the Illumina-derived GIS to correctly classify genomic instability status as determined by the Myriad test (cutoff, 42) was evaluated using area under the receiver operating characteristic (AUROC).

Result(s)* Agreement rates are reported in the Table. The GIS correlation between the 2 tests was 0.980 (all samples) and 0.975 (non-BRCAm cohort). AUROC was 0.992 (all samples) and 0.988 (non-BRCAm cohort). Prevalence (Illumina and Myriad tests) was $51.0 \%$ and $49.2 \%$ for overall HRD and $27.6 \%$ and $25.5 \%$ for BRCAm. Success rates (Illumina and Myriad tests) were 86.8\% (197/227) and 94.1\% (239/254) (overall HRD), 88.1\% (200/227) and 97.6\% (248/254) (BRCAm), and 91.2\% (207/227) and 94.1\% (239/254) (GIS); after re-running the failed samples with the Illumina test, rates were $90.3 \%, 92.5 \%$, and $93.4 \%$, respectively.
Abstract 978 Table 1 Agreement rates for the illumina test versus the myriad test

\begin{tabular}{|c|c|c|c|}
\hline & $\begin{array}{l}\text { Positive Percentage } \\
\text { Agreement, \% } \\
(95 \% \mathrm{Cl})\end{array}$ & $\begin{array}{l}\text { Negative Percentage } \\
\text { Agreement, \% } \\
(95 \% \mathrm{Cl})\end{array}$ & $\begin{array}{c}\text { Overall } \\
\text { Percentage } \\
\text { Agreement, \% } \\
(95 \% \mathrm{Cl})\end{array}$ \\
\hline $\begin{array}{l}\text { HRD } \\
\text { (positive vs negative) } \\
(\mathrm{N}=194)\end{array}$ & $92.3(85.6-96.1)$ & $96.7(90.7-98.9)$ & $94.3(90.1-96.8)$ \\
\hline $\begin{array}{l}\text { BRCAm } \\
\text { (positive vs negative) } \\
\text { (N=197) }\end{array}$ & $92.9(83.0-97.2)$ & $98.6(95.0-99.6)$ & $96.9(93.5-98.6)$ \\
\hline $\begin{array}{l}\text { GIS } \\
(<42 \text { vs } \geq 42) \\
(\mathrm{N}=204)\end{array}$ & $91.3(84.2-95.3)$ & $98.0(93.1-99.5)$ & $94.6(90.6-97.0)$ \\
\hline
\end{tabular}

Conclusion* Illumina test and Myriad test HRD, BRCAm, and GIS detection results were in $>91 \%$ agreement. With both tests, GIS was highly correlated (0.98), and prevalence estimates of HRD and BRCAm rates were similar. Data suggest that a distributable solution such as the Illumina test may replicate the performance of the Myriad myChoice HRD assay.

\section{OLAPARIB IN COMBINATION WITH INHIBITORS OF ATR/ CHK1 PATHWAY LEADS TO INCREASED CELL DEATH IN OVARIAN CANCER CELLS SENSITIVE AND RESISTANT TO PARPI}

$Ł$ Biegała*, A Gajek, A Marczak, A Rogalska. Institute of Biophysics, Department of Medical Biophysics, Lodz, Poland

\subsection{6/ijgc-2021-ESG0.666}

Introduction/Background* Ovarian cancer (OC) is believed to be one of the most lethal gynaecologic malignancies worldwide. Despite advances in the treatment of OC after the introduction of poly(ADP-ribose) polymerase inhibitors (PARPi) in the frontline setting as maintenance therapy and in the recurrent setting, the 5-year survival rate of high-grade serous ovarian cancer (HGSOC) ranges between 35 and 40\%. PARPi exhibit meaningful activity against OC, however resistance to these agents emerges ultimately. Thus, there is a need to develop more effective treatments for OC. Recent reports highlighting increased OC cell reliance on ATR/CHK1 pathway gives hope to overcome PARPi resistance and prolong patient's survival.

Methodology The aim of this study was to estimate cytotoxic activity of PARPi (olaparib), the ataxia telangiectasia and Rad-3 related protein (ATR) inhibitor (ATRi, ceralasertib), and the checkpoint kinase 1 (CHK1) inhibitor (CHK1i, MK8776) alone or in combinations in PEO1 (BRCA2MUT) OC cell line, and in PEO1-derived olaparib-resistant (PEO1-OR) cell line developed by continuous incremental long-term treatment with olaparib. Here, we evaluated the effect of tested drugs on cell survival in respect of metabolic activity by MTT assay and colony forming capacity. We also preliminarily elucidated mechanisms conferring resistance to olaparib in OC cells by assessment of expression of key proteins (ATR, CHK1, PARP1, P-glycoprotein) by western blot analysis. Statistical analyses were performed using Student's t-test and ANOVA followed by the Tukey's multiple comparisons post-hoc test.

Result(s)* OC cells are more sensitive to combination of the drugs in comparison with monotherapy with each agent alone. What is more, treatment with single-agent PARPi or combination of PARPi/ATRi or PARPi/CHK1i activates ATR/CHK1 
pathway through increased phosphorylation of CHK1 on serine 345 in PEO1 olaparib-sensitive cells. Targeting PARP in combination with ATRi or CHK1i is also synergistic in olaparib-resistant OC cells. However, pCHK1 was upregulated after treatment with $\mathrm{CHK} 1 \mathrm{i}$ and PARPi/CHK1i in PEO1-OR cells. PARPi-resistance is associated with increased expression of PARP1, but not P-glycoprotein.

Conclusion* Our results show that olaparib in combination with ATRi or CHK1i prompts cell death of OC line sensitive and resistant to olaparib on the path of synthetic lethality.

This research was funded by the Polish National Science Centre, Poland (Project grant number: Sonata Bis 2019/34/E/ NZ7/00056).

\section{DIGITAL HEALTH-RELATED APP SUPPORTING PATIENTS WITH GYNAECOLOGICAL MALIGNANCIES: RESULTS OF A FEASIBILITY TRIAL}

J Wolff, I Stupin, I Sehouli*. Charité Campus Virchow Clinic, Frauenklinik, gynäkologische Onkologie, Berlin, Germany

\subsection{6/ijgc-2021-ESG0.667}

Introduction/Background* To investigate the feasibility and acceptance of the Mika health app by patients with cancer.

Methodology Prospective intra-individually controlled pilot study. Patients were randomly assigned to receive either digital psycho-oncological support in the form of the app or standard care.

Result(s)* A total of 70 patients were included, one-third of whom had high levels of psychological distress. Relative to the baseline during the 12-week period, the mean depression score decreased in the intervention group $(p=0.00078$ one-tailed, $p=0.0016$ two-tailed), without any changes in the control group. The most prevalent disease-specific side effect was fatigue, which decreased by $23.1 \%$ in the intervention group and by $31.1 \%$ in the chemotherapy subgroup $(p=0.02)$.

Conclusion* The digital app showed high acceptance and satisfaction among patients with gynaecological malignancies, regardless of age group.

\section{RESULTS OF NSGO-OV-UMB1/ENGOT-OV30 STUDY: A PHASE II STUDY OF DURVALUMAB AND OLECLUMAB IN PATIENTS WITH RELAPSED OVARIAN CANCER (OC)}

${ }^{1} \mathrm{M}$ Mirza*, ${ }^{2} \mathrm{JR}$ Henriksen, ${ }^{3} \mathrm{~J}$ Maenpaa, ${ }^{4} \mathrm{R}$ Depont Christensen, ${ }^{2} \mathrm{M}$ Waldstroem, ${ }^{5} \mathrm{~L}$ Tandaric, ${ }^{6} \mathrm{~K}$ Lindemann, ${ }^{7} \mathrm{H}$ Roed, ${ }^{3} \mathrm{~A}$ Auranen, ${ }^{8} \mathrm{~L}$ Akslen, ${ }^{9} \mathrm{M}$ Magnusson, ${ }^{9} \mathrm{~S}$ Lindberg, ${ }^{9} \mathrm{~K}$ Madsen, ${ }^{5} \mathrm{~L}$ Bjorge. ${ }^{1}$ Rigshospitalet, København, Denmark; ${ }^{2}$ Vejle University Hospital, Denmark; ${ }^{3}$ Tampere University Hospital, Finland; ${ }^{4}$ NSGO-CTU, København Ø, Denmark; ${ }^{5}$ Haukeland University Hospital, , Norway; ${ }^{6}$ Oslo university hospital, Norway; ${ }^{7}$ Rigshospitalet, Denmark; ${ }^{8}$ Haukeland University Hospital, Denmark; ${ }^{9}$ NSGO-CTU, Denmark

\subsection{6/ijgc-2021-ESG0.668}

Introduction/Background* The primary objective of this multicentre, phase II study was to evaluate the preliminary efficacy of O (3000mg, IV q2W) in combination with D (1500mg, IV, $\mathrm{q} 4 \mathrm{~W})$ in relapsed OC patients with the CD73 expression on intraepithelial inflammatory cells in the TME, defined by disease-control rate (DCR) at 16 weeks. Key eligibility criteria include: histologically confirmed OC with CD73 expression; relapsed disease; measurable disease; no prior immunotherapy; mandatory paired biopsy. 25 patients have been enrolled. Treatment was continued until disease progression, unacceptable toxicity, or withdrawal of consent. Primary endpoint is DCR; secondary endpoints include ORR, DoR, PFS, OS, safety and tolerability. Responses are also corelated to CD8 and PD-L1 positive expression in tumours. Assessment of PD-L1 $(\geq 5 \%)$ and CD8 $(\geq 5 \%)$ were performed by immunohistochemistry on archival tumour specimens. Clinical trial information: NCT03267589

Methodology The primary objective of this multicentre, phase II study was to evaluate the preliminary efficacy of $\mathrm{O}$ (3000mg, IV q2W) in combination with D (1500mg, IV, $\mathrm{q} 4 \mathrm{~W})$ in relapsed OC patients with the CD73 expression on intraepithelial inflammatory cells in the TME, defined by disease-control rate (DCR) at 16 weeks. Key eligibility criteria include: histologically confirmed OC with CD73 expression; relapsed disease; measurable disease; no prior immunotherapy; mandatory paired biopsy. 25 patients have been enrolled. Treatment was continued until disease progression, unacceptable toxicity, or withdrawal of consent. Primary endpoint is DCR; secondary endpoints include ORR, DoR, PFS, OS, safety and tolerability. Responses are also corelated to CD8 and PD-L1 positive expression in tumours. Assessment of PDL1 $(\geq 5 \%)$ and CD8 $(\geq 5 \%)$ were performed by immunohistochemistry on archival tumour specimens. Clinical trial information: NCT03267589

Result(s)* $80 \%$ of patients had received $\geq 2$ lines of therapy for relapse. DCR was 27\%, median PFS was 2.7 months and median OS was 8.4 months. $74 \%$ of patients were CD8-positive, $42 \%$ were PD-L1-positive, while $37 \%$ were both CD8 \& PD-L1-positive. Biomarker positivity was not significantly associated with better DCR $(p=0.584)$. TEAE: 1 thromboembolic event (grade 3), one neutropenia (grade 4), one cardiac arrest (grade5).

Conclusion* Combination of Oleclumab-durvalumab is feasible and demonstrate modest preliminary activity in relapsed OC. Further biomarker analysis research to predict response is ongoing.

\section{Vaginal and vulvar cancer}

\section{PRIMARY IMIQUIMOD TREATMENT VERSUS SURGERY FOR VULVAR INTRAEPITHELIAL NEOPLASIA - PITVIN STUDY. A RANDOMIZED CLINICAL TRIAL}

${ }^{1} \mathrm{G}$ Trutnovsky*, ${ }^{1} \mathrm{O}$ Reich, ${ }^{2} \mathrm{E}$ Joura, ${ }^{3} \mathrm{~A}$ Ciresa-König, ${ }^{3} \mathrm{~A}$ Widschwendter, ${ }^{4} \mathrm{C}$ Schauer ${ }^{5} \mathrm{G}$ Bogner, ${ }^{6 ; 7} \mathrm{Z}$ Jan, ${ }^{8} \mathrm{M}$ Holter, ${ }^{1} \mathrm{~K}$ Tamussino. ${ }^{1}$ Medical University of Graz, Obstetrics and Gynecology, Graz, Austria; ${ }^{2}$ Medical University of Vienna, Wien, Austria; ${ }^{3}$ Medical University of Innsbruck; ${ }^{4}$ Hospital BHB Graz; ${ }^{5}$ Medical University of Salzburg; ${ }^{6}$ Hospital Klagenfurt; ${ }^{7}$ Cancer centre Carinthia, Klinikum Klagenfurt, Klagenfurt, Austria; ${ }^{8}$ Medical University of Graz, Medical Statistics and Documentation, Graz, Austria

\subsection{6/ijgc-2021-ESG0.669}

Introduction/Background* Surgery is the standard treatment for HPV-associated high-grade vulvar intraepithelial neoplasia (HSIL/VIN), but recurrences occur in about 50\% of patients. Imiquimod, a topical immune response modifier, alters the local immune response in favour of HPV clearance and has been shown to be an effective treatment. The aim of this study was to compare the safety, effectiveness and acceptance of primary imiquimod treatment with surgical treatment of HSIL/VIN.

Methodology This was a multicentre randomised controlled trial of women with histologically confirmed HSIL/VIN. 